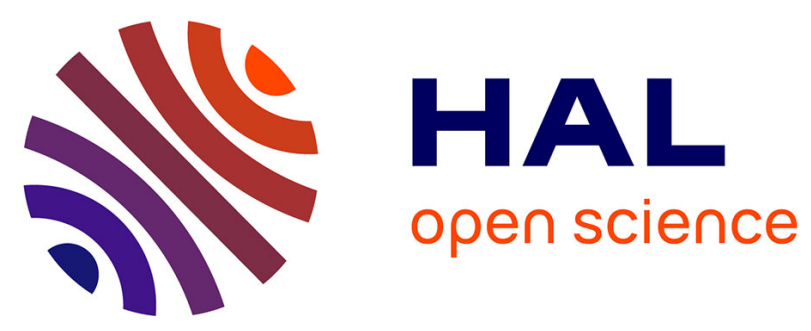

\title{
Revealing shape semantics from morphological similarities of a collection of architectural elements: The case study of the columns of Saint-Michel de Cuxa
}

David Lo Buglio, Vanessa Lardinois, Livio de Luca

\section{- To cite this version:}

David Lo Buglio, Vanessa Lardinois, Livio de Luca. Revealing shape semantics from morphological similarities of a collection of architectural elements: The case study of the columns of Saint-Michel de Cuxa. Digital Heritage International Congress DH'13, Oct 2013, Marseille, France. pp.465-472, 10.1109/DigitalHeritage.2013.6743785 . hal-02336914

\section{HAL Id: hal-02336914 https://hal.science/hal-02336914}

Submitted on 5 Nov 2019

HAL is a multi-disciplinary open access archive for the deposit and dissemination of scientific research documents, whether they are published or not. The documents may come from teaching and research institutions in France or abroad, or from public or private research centers.
L'archive ouverte pluridisciplinaire HAL, est destinée au dépôt et à la diffusion de documents scientifiques de niveau recherche, publiés ou non, émanant des établissements d'enseignement et de recherche français ou étrangers, des laboratoires publics ou privés. 


\title{
Revealing shape semantics from morphological similarities of a collection of architectural elements
}

\author{
The case study of the columns of Saint-Michel de Cuxa
}

\author{
David LO BUGLIO ${ }^{1,2}$, Vanessa LARDINOIS ${ }^{1} \&$ Livio \\ $\mathrm{DE} \mathrm{LUCA}^{1}$ \\ ${ }^{1}$ UMR 3495 CNRS / MCC MAP Gamsau \\ Marseille, France \\ $\{$ david.lobuglio, vanessa.lardinois, \\ livio.deluca\}@gamsau.map.archi.fr
}

\begin{abstract}
Over the last three decades, the introduction of digital technologies in the field of architectural documentation has profoundly changed the tools and the acquisition techniques. Most of the developments concerns metrical and colorimetric characteristics of the objects studied. These developments, surrounding the practice of architectural survey, tend to respond primarily to the requirements of completeness. In this context, it seems necessary to assess the impact of these instruments on the cognitive value of architectural representation. With a strong technological presence, the study of the built heritage is facing a problem of "overload" of information. Indeed, it fails to strengthen the representation in its role as vehicle of knowledge. Confronted with the intelligibility deficit, this article propose an original approach for reading morphological features of an artifact by using a bottom-up approach: the meaning of the elements comes from the analysis of low-level geometric properties of a collection of instances related to the same theorical model. The idea is to rely on data accumulation in order to make emerge high-level semantic features from the comparative analysis of common low-level geometric features. The introduced principles are illustrated by the comparative analysis of 31 columns of the cloister of the abbey of Saint-Michel Cuxa, aimed to identify a common semantic layout.
\end{abstract}

Keywords-Architectural representation, morphological analysis, semantic characterization, image Processing.

\section{INTRODUCTION}

Today, it seems that the three-dimensional representation is an effective medium for the documentation, the study and the heritage enhancement. In this context many research projects focus on tools and methods of architectural digitization. But, beyond the technological impact, the understanding of their contribution for the production and the cognitive value of representations constitute a major challenge for research.

Over the past three decades, the introduction of digital technologies in the field of architectural documentation has profoundly changed the instruments and techniques of acquisition. These developments have increased the accuracy and completeness of architectural survey. The discrimination of information provided by the operator doesn't seem to be decisive anymore in a survey process based on quantitative requirements [1]. Whether it is the lasergrammetry or the photogrammetry, all the techniques being developed for

\author{
${ }^{2}$ Faculté d'Architecture (La Cambre Horta), ULB \\ Bruxelles, Belgium \\ david.lo.buglio@ulb.ac.be
}

heritage documentation tend to respond primarily to the completeness requirements.

If there is a desire to use the digital model for structuring, organizing and archiving heterogeneous information, the threedimensional representations of buildings are mostly exploited as a means to document the existing state of an object. By continually pushing the level of completeness of the digital models, nowadays one can observe a strong metric and visual coherence between architectural artifacts surveyed and their digital instances.

However, the growing body of point clouds, 3D models or un-interpreted data does not seem to increase the level of intelligibility or the cognitive dimension of the representations produced. For example, human analysis is still crucial to the understanding of the different conceptual articulations of an architectural element. Faced with this overload of "information" and the intelligibility deficit resulting, it is necessary to provide a methodological answer to articulate, within the same graphical space, the concepts of "multiplicity" and "intelligibility".

Today, the majority of responses to this issue are based on a "top-down" approach, i.e. an approach that uses the prestructured knowledge of the domain [2]. Although there are several support tools for the organization, the segmentation and the semantic characterization of $3 \mathrm{D}$ data, most of these solutions depend strongly on the level of the operator's understanding of the object [3]. One of the challenges of this research is to provide an alternative to the traditional approaches (subjective) with the aim to offer (objective) analysis solutions to increase the cognitive value of representations. So, this paper explores how the statistical study and the data accumulation allow achieving an objective estimation of the morphological properties of an architectural object. In line with this ambition, the second challenge is to evaluate how the creation of a morphological signature (common to a set of elements) can be used to the shape classification. Through the study of the columns of the cloister of the abbey of Saint-Michel Cuxa, we will assess what the examination of morphological similarities can bring to the semantic characterization of architectural elements. 
By focusing on the epistemological issues surrounding the survey and the representation of architectural artifacts, this article tries to:

- locate issues related to the intelligibility deficit.

- mention some approaches implemented to answer at this first issue. It should be noted that these approaches focus mainly on a description "from above" (topdown), ie a description that takes advantage of the prestructured knowledge of the domain.

- $\quad$ propose an alternative to these approaches by focusing on the possibilities of a "bottom-up" study which relies on the "objective" estimation of the morphological characteristics of an object.

- finally, evaluate the relevance of this new approach from the results obtained on a specific case study: the columns of the cloister of the Abbey of Saint-Michel Cuxa.

\section{BETWEEN COMPLEXITY AND INTELLIGIBILITY OF ARCHITECTURAL REPRESENTATIONS}

With their ability to combine within the same graphical space the architectural codification and the figuration of the object, the canonical representation systems of architecture (plan, section, elevation and perspective) are, since the Italian Renaissance, the privileged vehicle of the shape knowledge. Nevertheless, this paradigm of communication, common to all disciplines concerned with the study of built heritage, is implicitly challenged by the arrival of digital technologies [4].

Indeed, many tools and methods in the field of architectural documentation have been modified by the introduction of digital solutions. The architectural photogrammetry has reached a level where access and cost are able to fill many needs at the scale of the built heritage. The development of multi-stereo correlation systems allows registration of dimensional and colorimetrical properties. Therefore, It is no longer uncommon to get scans describing the architectural object with millions of colored points (coordinates) [5].

If this technology craze has helped to strengthen the architectural survey, it did not consolidate the intrinsic cognitive dimension to its practice. Its analytical value is mainly due to the work of interpretation and at the choice of information collected on the object. In a context where the level of visual and metric coherence between the object and its digital instance continues to grow, the mass of un-interpreted data raises questions. Is the multiplicity of acquired data is able to increase the level of intelligibility of architectural representation?

A study published in 2011, on the cognitive dimension of architectural surveying, offers an observation of the intelligibility level of a set of representations depending on the degree of automation of the tools and methods mobilized [6]. We mean with "intelligibility", the ability of the 3D digitization to refer to a world of knowledge. One understands that the informative richness of digitization is partly linked to the cognitive engagement of the operator and not the amount of data they contain. In order to achieve representations with a high degree of intelligibility, the action of the operator seems crucial for the analysis, classification and interpretation of data.

Current approaches (or automated process) effectively guarantee multiple data processing (color and geometry) without improving the intelligibility of representations produced. In fact, they respond only partially to the needs of communities concerned with the morphological analysis of the building heritage [7]. In this context, the only research on the development of tools for the acquisition and data processing cannot respond structurally to the intelligibility deficit observed. It should be noted that this criticism is not intended to call into question the relevance of technological advances in the field of architectural representation but to understand their limits and deficits.

The main issue of this work is whether the introduction of a new methodology for shape analysis and statistical analysis would permit an approach where the data flow would support the intelligibility of representations produced. Thus, this paper presents an approach to complete the acquisition protocols. This research, based on the use of multiple data, aims to produce a semantic description from a low level morphological analysis.

\section{FROM THE HIGH LEVEL SEMANTIC DESCRIPTION TO THE LOW LEVEL GEOMETRIC DESCRIPTION}

For some years now, the communities involved in the heritage documentation seem gradually take over the problems related to intelligibility deficit. There are now numerous studies concerned with the exploitation of non-interpreted data. Indeed, the geometric description and the analysis of architectural shapes require a reduction of multiplicity to intelligibility. Nowadays, there are several applications for the understanding of geometric shapes. Research around the automatic and semi-automatic segmentation, the semantic annotation, the parametric modeling or the image-based modeling from geometric primitives is symptomatic of the need to organize the $3 \mathrm{D}$ digitization around structured knowledge [8] [9] [10].

Thus, some recent research provides satisfactory answers to the problems of intelligibility. Most of them can be considered as a "top-down" approach because the operator leads the analysis from the top by taking advantages of the prestructured knowledge of the domain. This approach requires a semantic enrichment process where human analysis is decisive for the steps of segmentation, 3D reconstruction and / or semantic annotation.

It was also at the heart of two relevant research approaches. The first one focused on the formalization of moldings based on the rules governing the combination of geometric atoms [11]. This is a formalization of high level since the rules emanate from an architectural semantics. The second one focused on the development of a parametric modeling tool (Generative Modeling Language) for the formalization of architectural concepts from a low-level geometric vocabulary. In this case, it is a lower level since the reconstruction articulates a geometric semantics [12]. 
If these two examples differ in their analysis scale, architectural semantics on one side and geometric semantic on the other side, they both rely on a grammar of shapes. Therefore, we can consider that the two works offer a "topdown" approach. But beyond this first orientation, it seems necessary to complete this first step with an approach that would take advantage of the multiplicity of acquired data. This implies that the meaning of the elements would come from a "free" morphological estimation and not from a shape thesaurus. The "free" estimation corresponds to the opportunity to study without bias on the results, the point aggregate, the curvatures of surfaces and volumes of the architectural elements.

Beyond the study of singular elements, the use of statistical analysis would permit to find correspondences between different geometric entities. Morphological comparisons that can be done on a set of shapes offer opportunities to study similarities in a collection of architectural elements. Indeed, by establishing common geometric observation criteria and multiplying comparisons between elements, it is possible to identify morphological signatures that can contribute, in a second step, at the semantic characterization of a collection. This approach is therefore based on the self-expansion of a large amount of data.

The medium-term challenges of this approach are:

1. to establish new shape classification criteria. The comparison of architectural objects on the basis of geometric similarities aims to create libraries of architectural shapes organized around pre-structured knowledge.

2. to question architectural treaties with the classification work (through the geometrical analysis of the typical shapes, the composition rules, the principles of scale, the positioning constraints, the orientation, etc...).

3. to study the variability of architectural elements. In the field of built heritage, the morphological signature (or theoretical model) of a collection of elements can serve as the basis for the study of their variability in space and time. In addition, it can also have an impact on the understanding of the evolution of a style. In the continuation, the use of self-extension mechanisms can provide useful answers to observe unclassified and uninterpreted shapes.

\section{LOW LEVEL DESCRIPTION : THE CASE OF THE COLUMNS OF SAINT-MICHEL DE CUXA}

In order to experiment this approach, a first study was conducted on the columns of the cloister of the abbey of SaintMichel Cuxa. They have the dual advantage of being in a good state of conservation and belong to a group composed of 31 columns present on site. Indeed, the "bottom-up" approach is only relevant if it is part of a cumulative study. Therefore the challenge is to assess the common denominators / geometric similarities in the columns collection. It is also interesting to note that the many theories that have existed since the Renaissance make the column a relevant case study [13].
The identification of a morphological signature could lead us towards a common theoretical model to all of the columns of the cloister. It could emerge a 32nd theoretical column from the 31 columns that still exist. It may be recalled that this approach aims to assist human analysis on the shapes classification and comparison.

The survey (used in this study) combines the latest representation systems with the architectural image-based modeling developments. Indeed, at the level of architectural elements documentation, image-based modeling (survey, 3D modeling and representation from images) has reached a point where costs, accessibility and quality of results can fill many needs at the built scale. The columns have been digitized during a photogrammetric survey campaign in December 2010. The digital instances of each column (obtained after the acquisition on site and the processing of the different point clouds) express a certain level of completeness "Fig.1". But beyond a high geometric fidelity, our concern is the degree of intelligibility of the digitization, and their ability to build a theoretical model.

Before describing the technical principles of the approach (and the establishment of a first morphological signature), we will observe the tools currently available at the level of shape analysis. Thus, this chapter will focus on:

- the conversion of 3D digitization into a twodimensional representation (most suitable for image analysis).

- the existing algorithms in the field of image analysis and thus on the relevant shape descriptors for the creation of morphological signature.

- the study of the major geometric discontinuities of each column, the evaluation of their average and their
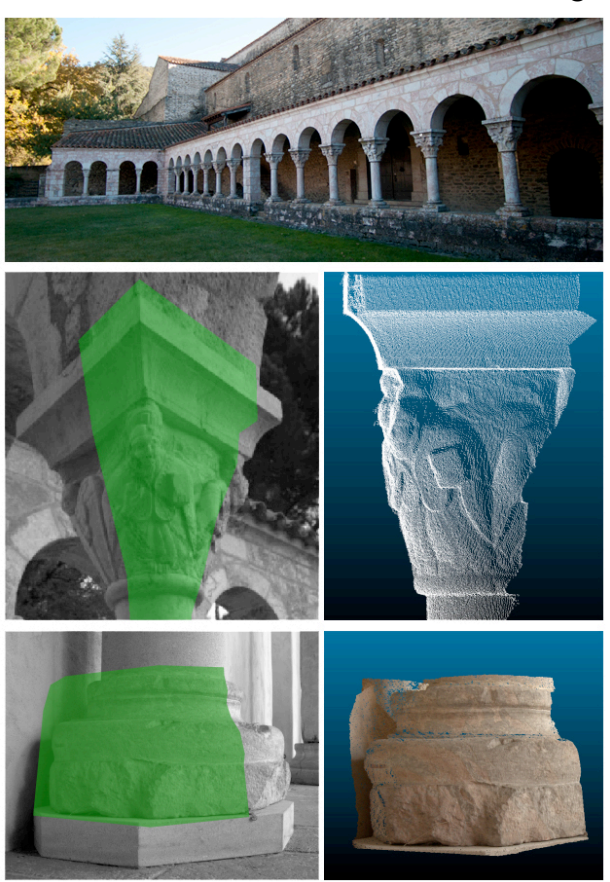

Fig. 1. Masks and point clouds from the photogrammetric acquisition of the columns of Saint-Michel de Cuxa 
re-projection in the form of relevant profiles (in order to build a first morphological signature).

- the link between each digital instance and its theoretical model.

\section{A. Acquisition and extraction of depth maps}

In order to use existing tools in the field of image analysis, one of the issues is to convert in two dimensions the complex geometric articulations of the columns. The polygonal models of each column are projected onto a two-dimensional depth map. This map is obtained from the projection of the polygonal mesh on a UV cylinder surrounding it. The use of a cylinder is not intended to characterize the geometric complexity of the object. The ambition is to refer to the concept of the column by exploiting its geometric archetype "Fig.2".

The interest of the approach is that each pixel of the cylinder is encoded as a grayscale value determined from the distance separating it from the polygonal mesh. The unfolded projection cylinder (or the two-dimensional depth map) contains all the geometric complexity of the digital instance. From this map or from other computed maps (like the normal map and the ambient occlusion map), it is therefore possible to lead a precise morphological study based on image analysis tools [14].

\section{B. Morphological analysis and description of low level}

At the level of shapes study, image analysis algorithms offer many opportunities for contour extraction, identification of lines and analysis of similar regions within an image [15]. To understand the benefits and the limitations of these tools for the low level morphological study, all the known solutions have been systematically experimented and rigorously inventoried.

If, some algorithms are an appropriate solution for image analysis in the industrial or the medical field, their diversion for the study of heritage artifacts presents some difficulties. They require a hard work of setting and adaptation to meet specificities of built (automatic identification of rhythms, geometric discontinuities, similarities,...).

A first experiment has permitted to assess some shape analysis operators directly on the depth maps coming from the columns "Fig.3". It is important to note that the operators are estimated on their contribution to the low level geometric description, e.g. on their ability to identify some geometric breaks in a column. The three families of description methods and image process methods mobilized were:

- Threshold-based segmentation

\section{- Contour-based segmentation}

- Region-based segmentation

Nevertheless, the analysis operators only make sense if they can isolate, bring together or discriminate some of information within the image. We mean by relevant descriptors, those able to locate specific morphological characteristic (scale variation, symmetry, degree of curvature, surface discontinuity,...).

Before going further, it is important to remember that the description of the vast majority of shapes can be done by the knowledge of:

1. the geometry, which refers to the understanding of volumes and surfaces of the object

2. the topology, which focuses on neighborhood relations within a shape

3. and finally, the organization or the spatial composition of the basic physical entities

These three points are an appropriate observation triangle at different levels of granularity (scale). Since our interest is on the entity as a whole (column), this work focuses primarily on the geometric level. This level involves the characterization of the morphology of an element according to two aspects: its curves and its discontinuities.

Indeed, the geometric study of a shape implies the knowledge of its curves. This is explained by the fact that any surface can be defined by " $n$ " curves. For each one corresponds a unique profile whose mathematical representation is similar to a set of functions. The calculation of curvature maps is therefore an essential aspect for the understanding the surfaces [16].

But beyond this first aspect, a shape can also be described by its major discontinuities. Is meant by major discontinuities, each surface change marked by the rupture of a curvature. In the particular case of the study of architectural elements, the geometrical discontinuities provide the understanding of the conceptual articulations present in the object. Each passage of a concept to another (e.g. from the shaft of the column up to the astragalus) results in a strong discontinuity of surface. Considering the interest that can represent this last aspect for the communities involved in the study of the built heritage, the rest of this chapter is primarily dedicated to the results obtained through the study of geometric discontinuities.

If this paper focuses, up to now, on the study of singular elements, it is necessary to remember that the final goal of this research is to achieve a common morphological signature to the 31 columns. 

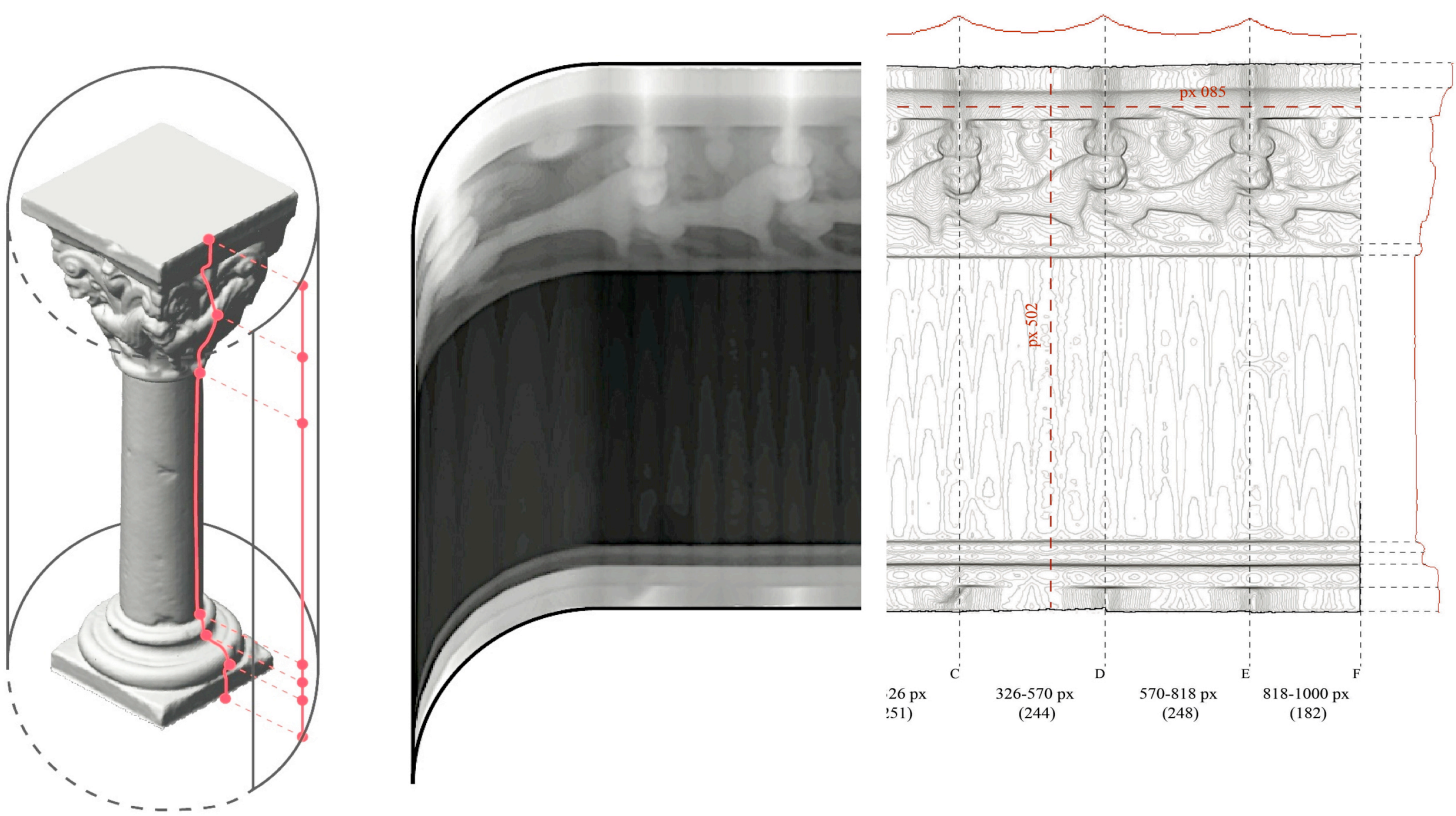

Fig. 2. Generating a depth map from the polygonal mesh, analysis of geometric discontinuities and profiles from the map.

\section{Study of morphological discontinuities and $3 D$ reprojection of the average profiles}

To summarize, the general issue of this work is to provide a response to the lack of intelligibility mentioned before, a deficit that the technological developments do not solve. Rather than consider the enrichment of representations by a "top-down" approach, the method proposed here is based on the accumulation of data. Through the accumulation, the idea is to bring out common geometric characteristics of to a set of architectural elements. In this case, our attention focuses on the study and on the exploitation of discontinuities with the aim to establish a first common morphological signature of the columns. Its implementation is organized around a

\section{development in three stages:}

\section{1) First time: line detection}

The observed image analysis tools provide useful technical tracks for the implementation of shape descriptors. Indeed, the study of discontinuities requires to locate and isolate significant lines present in the depth maps. They correspond to abrupt changes between shades of gray on a portion of the image.
Among the operators for the contour-based segmentation, the standard Hough transform (SHT) is a pattern recognition technique to automatically detect the horizontal and vertical lines present in the image.

In order to optimize the detection operator, it is preferable, in a first step, to apply a contour detection like the type Sobel [17]. It provides a much lighter image with less detail. The contours are sharper and the shapes are properly closed. The threshold value is adapted to obtain an optimal display of contours. This pre-treatment is necessary because the Hough operator is preferably applied on binary images.

At the level of the lines detection operator, the general principle of the Standard Hough Transform is to admit the existence of an infinite number of lines passing through all the points of the image. Each segment can be characterized with two parameters: an angle $\theta$ (Theta) and a length $\rho$ (rho) perpendicular to the line, and passing through the origin of the system. The lengths and the angles obtained in the Cartesian system $(\mathrm{x}, \mathrm{y})$ are then represented in the form of coordinate vectors RHO-THETA in Hough space. They are (in this space) sinusoidal curves that correspond to each point of the image.
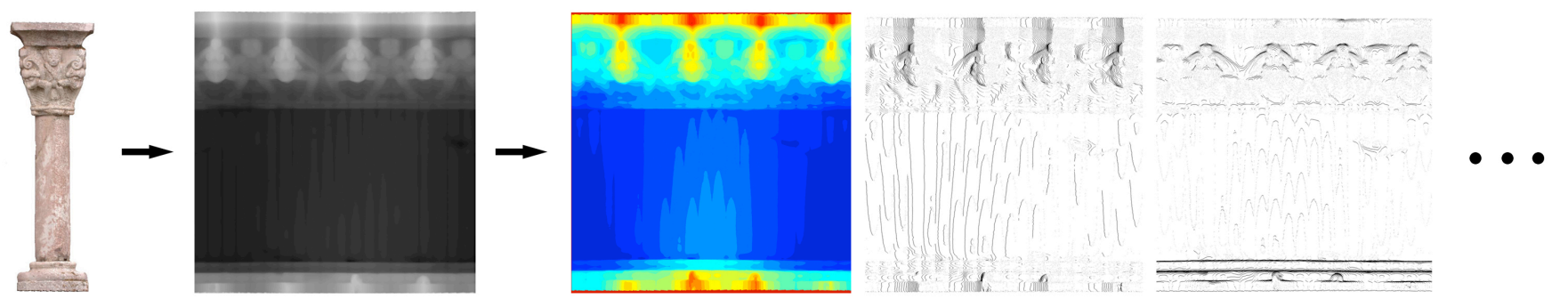

Fig. 3. Threshold-based segmentation and contour-based segmentation on the depth map from the west column No. 25. 
The intersection of two curves marks the linear link between two points in the Cartesian system [18].

Due to its use on architectural elements, sometimes strongly altered, it may happen that the Hough transform fails to correctly identify some lines of the image. Although the majority of the line segments detected are horizontal, the transform also recognizes oblique lines (not adapted for future use). Solutions of discrimination have been created in order to correct these errors of interpretation of the Hough transform. Some oblique (whose inclination does not exceed a discriminator value) are adjusted to obtain usable horizontal, i.e. a unique coordinate " $y$ " for the discontinuity. This adaptation is necessary to meet the requirements of the morphological study of the column.

As presented, the Standard Hough Transform has been adapted and configured in order to automatically bring out all the linear networks present in the depth maps. These networks correspond to the visible representation of the major geometric discontinuities (both horizontal and vertical) of the object. The analysis of low-level (or bottom-up), as seen here, seems to provide a satisfactory response to the automatic detection of conceptual articulations present in the column.

The observation of the results shows that all the discontinuities detected actually correspond to the transition between two architectural concepts. One recognizes in the illustrations below "Fig.4", the transitions between:

1. the plinth and the lower torus

2. the lower and upper torus

3. the upper torus and the shaft

4. the shaft and the astragalus

5. the astragalus and the historiated capitals

6. the historiated capitals and the echinus

7. the echinus and the abacus
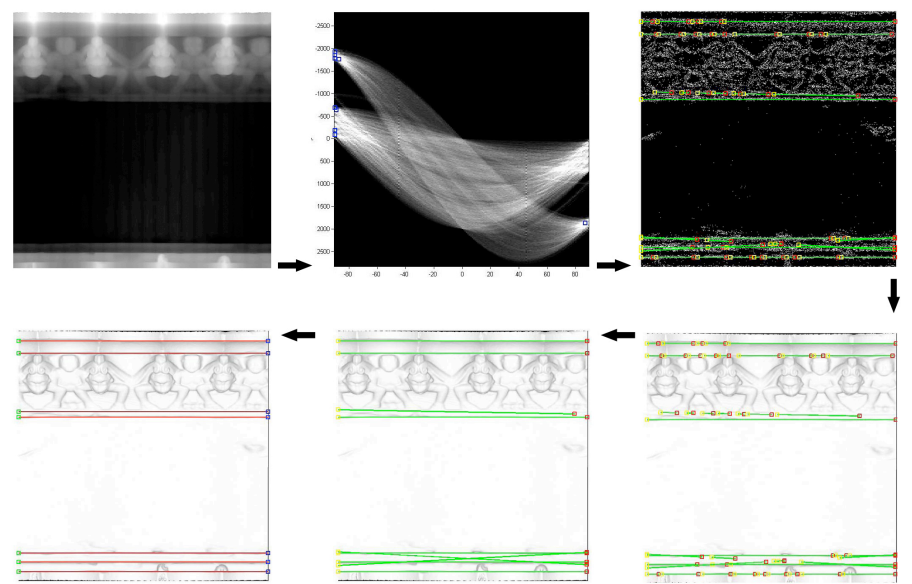

Fig. 4. Depth map, sinusoidal of the Hough transform, detection of image lines, extrapolation of lines and discrimination of certain oblique, view of the mean value (on "y").
2) Second step: extraction and $3 D$ reprojection of the relevant profiles

Before studying the average value whose aim is the creation of a morphological signature common to all the columns, this point focuses on the exploitation of the discontinuities at the level of the object. Although it is possible at this stage to conduct an analysis (for each depth map) on the heights and the distances between the discontinuities, it is also possible to achieve the simplified geometric reconstruction of each 31 columns. Indeed, each discontinuity on the map corresponds to the position of a relevant profile for the simplified formalization of the object.

These profiles can be obtained by the projection of the gray values of each pixel located along the discontinuity. By considering that these shades are the graphical representation of the distances separating the digital instance from the cylinder of projection (see above), this implies that all the pixels contain the information of their position. These values (coded from 0 to 255) are first converted into a metric scale and then projected perpendicularly to the plane of the depth map (here along the $\mathrm{Z}$ axis) "Fig.5".

To find similar profiles of a column section, it is necessary to transfer the result of the "planar" projection on a "cylindrical" projection. To solve this problem, the profiles obtained in a Cartesian coordinate system are transposed in a polar coordinate system where the value of each point in $\mathrm{Z}$ corresponds to the radius $(\rho)$ of the new profile and the value $X$ to the radians $(\theta)$.

With this proposal, we can already consider the analysis of profiles and discontinuities as a relevant way to describe certain geometric characteristics of the column (e.g. rhythm, minimums, maximums and average).

\section{3) Third step: study of average values}

If the previous step provides interesting avenues for the reconstruction of simplified geometries, the study of average offers opportunities for the creation of morphological signatures. It is conceivable that the "hybridization" of the values of discontinuities (from a collection of columns) can bring out the generic characteristics of the shape. In this perspective, this point focuses on the creation of a morphological signature based on the geometric discontinuities of the 31 columns processed.

One might think that the average calculation of all the depth maps of the 31 columns may be sufficient to formalize a valid general average. However, such an approach would lead to the construction of a 32nd theoretical column shapeless, unable to guarantee the intelligibility of its various articulations.

To bypass the geometric aberration that would result, it is possible to separate the study of average in three distinct parts:

1. the study of the average number of discontinuities observed for each depth map,

2. the study of the average vertical position of discontinuities for each of the 31 columns,

3. the study of the average gray values between discontinuities of the same level (i.e. between discontinuities in the same place on different maps). 

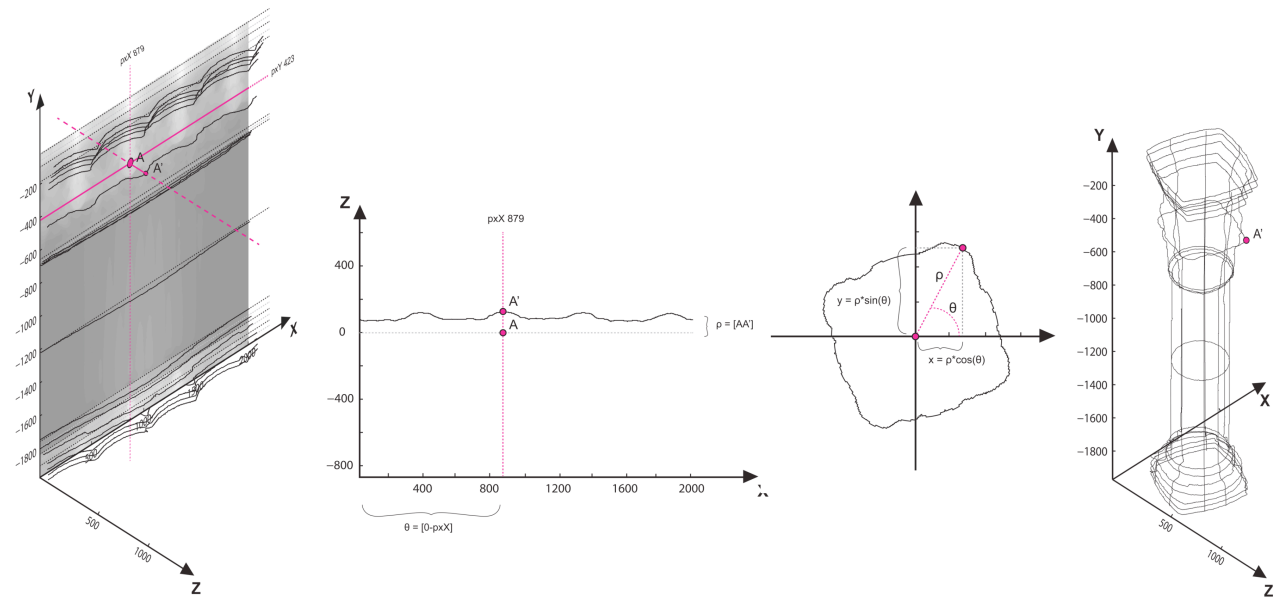

$$
\begin{aligned}
\rho=\left[\mathrm{AA}^{\prime}\right] ; \theta & =(2 \pi / \text { length }(\mathrm{X})) * \operatorname{pxX} \\
\mathrm{y} & =\rho * \operatorname{SIN}(\theta) \\
\mathrm{x} & =\rho * \operatorname{COS}(\theta)
\end{aligned}
$$

Fig. 5. 3D reprojection of the discontinuities.

These different steps in the analysis have been implemented in a tool that is based on the developments of the previous point "Fig.6". The mean values (in the form of profiles) are projected in 3D in order to represent graphically a first morphological signature. It is interesting to note that the theoretical model developed expresses all conceptual articulations present in the majority of the columns. The observation of all the columns shows that some of them have altered parts. For example, the column $n^{\circ} 24$ of the west wing has a heavily damaged base (especially at the level of the plinth). But with the accumulation of data, the degradation (even important) does not appear in the theoretical model. Although it is only the representation of the average discontinuities, the morphological signature described a first common semantic skeleton for all the columns, the $32 \mathrm{nd}$ theoretical column.

\section{CONCLUSIONS AND PERSPECTIVES}

In a context where the architectural surveying is dependent on tools and technologies that it mobilizes, the heritage documentation is facing a problem of "overload" of information. Once collected, they fail to strengthen the representation as a vehicle of knowledge. In front of the intelligibility deficit, this research has provided methodological orientation based on a "bottom-up" approach. The semantic characterization based on morphological similarities of elements offers new opportunities for the communities concerned with the study of built heritage. This work also shows that the enrichment of representations with prestructured knowledge of the domain could be complemented by the presence of a geometric description based on the study of similarities.
As we could see, the depth maps are an ideal medium for the analysis of discontinuities in a column. By their ability to highlight the conceptual articulations, these breaks help to ensure the reconstruction of simplified geometries, geometries that maintain their level of intelligibility. But beyond these aspects, one also observed that the calculation of averages constitutes an interest for the definition of morphological signatures. It opens avenues for the formalization of a $32 \mathrm{nd}$ theoretical column which interest is to serve as a reference for the study of altered elements.

However, one of the current limitations of this research is the lack of shape descriptors taken into account for the creation of this first morphological signature. In the current state of this work, the descriptors focus exclusively on the study of discontinuities. An answer would be to include other shape attributes. For example, one can think of the study of lines of curvature on surfaces.

In conclusion, the possibility of linking the concepts of multiplicity and intelligibility opens many tracks. If the simplified reconstruction of complex geometries shows interesting avenues for the field of 3D modeling, the formalization of morphological signatures constitute also an important issue. Their use could meet the following challenges:

- the visualization of the gap between the digital instance and the theoretical model (in order to better understand the alterations of buildings).

- the study of similarities between objects. The idea is to compare their discontinuities to find geometric correspondences in a collection of blocks, for example. This would permit to support the research in archeology, especially for the anastylosis work. 


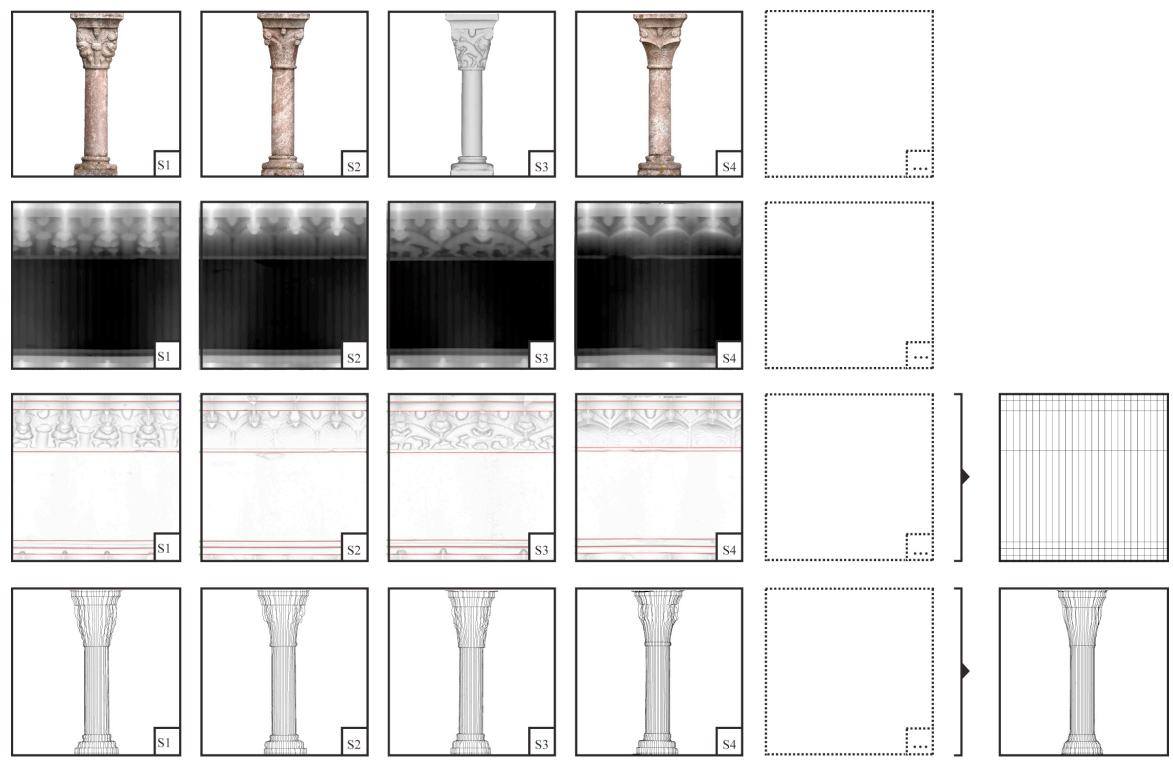

Fig. 6. First 4 columns of the collection: Polygonal mesh, depth map, discontinuities, simplified geometric reconstruction and average values (right side).

- the classification of shapes according to low level morphological criteria. The use of theoretical models could provide an opportunity to better understand the propagation of an architectural style in time and space. This last point makes legitimate the reformulation of shape thesaurus in the light of morphological signatures.

Finally, to conclude, this approach avoids the failings of the representation of the territory 1:1 scale that Borges evoked [19], where the practice of survey is reduced to the faithful reproduction of the visual attributes of space.

\section{REFERENCES}

[1] Collectif. Le Relevé En Architecture Ou L'éternelle Quête Du Vrai. Lieux Dits, 2011.

[2] A. Treisman, and G. Gelade. "A Feature-integration Theory of Attention.” In Cognitive Psychology 12, n¹, 1980, pp. 97-136.

[3] S. Spina, K. Debattista, K. Bugeja, and A. Chalmers, "Point Cloud Segmentation for Cultural Heritage Sites.” In Vast 2011. The 12th VAST International Symposium on Virtual Reality, Archaeology and Cultural Heritage, Prato: Eurographics press, 2011, pp. 41-48.

[4] D. Mediati, L'occhio sul mondo per una semiotica del punto di vista. Soveria Mannelli: Rubbettino, 2008.

[5] M. Pierrot-Deseilligny, L. De Luca, and F. Remondino. "Automated Image-Based Procedures for Accurate Artifacts 3D Modeling and Orthoimage." In XXIIIth International CIPA Symposium, 2011.

[6] D. Lo Buglio, and L. De Luca. "Critical Review of 3D Digitization Methods and Techniques Applied to the Field of Architectural Heritage: Methodological and Cognitive Issues." In Vast 2011. The 12th International Symposium on Virtual Reality, Archaeology and Cultural Heritage, Prato: Eurographics press, 2011, pp.5-12.

[7] J-Y. Blaise, and I. Dudek. "Informative Modelling." In MIA Journal, no. 1, 2006, pp. 143-154.
[8] G. Oxholm, and K. Nishino. "Reassembling Thin Artifacts of Unknown Geometry." In Vast 2011. The 12th VAST International Symposium on Virtual Reality, Archaeology and Cultural Heritage, Prato: Eurographics press, 2011, pp. 49-56.

[9] A. García Castañeda, B. Brown, S. Rusinkiewicz, T. Funkhouser, and T. Weyrich. "Global Consistency in the Automatic Assembly of Fragmented Artefacts." In Vast 2011. The 12th VAST International Symposium on Virtual Reality, Archaeology and Cultural Heritage, Prato: Eurographics press, 2011, pp. 73-80.

[10] L. De Luca, La photomodélisation architecturale. Relevé, modélisation et représentation d'édifices à partir de photographies, Eyrolles, 2009.

[11] L. De Luca, P. Véron, and M. Florenzano. "A Generic Formalism for the Semantic Modeling and Representation of Architectural Elements." In The Visual Computer 23 n³, 2007, pp. 181-205.

[12] S. Havemann, and D. Fellner. "Generative Parametric Design of Gothic Window Tracery." In Shape Modeling International, 2004, pp. 350-353.

[13] A. Palladio, The Four Books of Architecture. New York: Dover Publications, 1965.

[14] P. Papadakis, I. Pratikakis, T. Theoharis, and S. Perantonis, "Panorama: A 3D Shape Descriptor Based on Panoramic Views for Unsupervised 3D Object Retrieval.” In International Journal of Computer Vision 89, no. 2-3, 2010, pp. 177-192.

[15] D. Zhang, and G. Lu. "Review of Shape Representation Anddescription Techniques." In Pattern Recognition, Elsevier edition, 2004.

[16] L. De Floriani, and M. Spagnuolo. Shape Analysis and Structuring. Springer, 2007.

[17] B. Jähne, H. Scharr, and S. Körkel. "Principles of Filter Design." In Handbook of Computer Vision and Applications. Academic Press, 1999.

[18] R. Duda, and P. Hart. "Use of the Hough Transformation to Detect Lines and Curves in Pictures." In ACM 15, no. 1, 1972, pp. 11-15.

[19] J. Borges, "De la rigueur de la science." In L'auteur et autres textes, Paris: Gallimard, 1982, p.221. 